\title{
Advances in 5-axis CNC laser machining system
}

\author{
Yongquan Zhou ${ }^{1, a,} *$, Songling Zhang ${ }^{2, b}$, Haibing Xiao ${ }^{1, c}$, Zhang Wei ${ }^{1, d}$ \\ ${ }^{1}$ School of Mechanical \& Electrical Engineering, Shenzhen Information Institute of Technology , China \\ ${ }^{2}$ Hymson Laser Co. Ltd, Shenzhen, China \\ a zhouyq@sziit.edu.cn, ${ }^{\mathrm{b}}$ bill.zhang@hymson.com, ${ }^{\mathrm{c}}$ Xiaohb@sziit.edu.cn, ${ }^{\mathrm{d}}$ Zhangw@sziit.edu.cn \\ *corresponding author
}

Keyword: 5-axis CNC laser system, Texture mapping,3D texture model, High curvature surface.

\begin{abstract}
We present a novel method of laser machining 3D curved surface by 5-axis CNC laser machining system pertaining to the manufacturing processes of laser marking, laser mold texturing and laser direct structuring (LDS). We begin by presenting the need of texture mapping technology which is used in 3D curved surface for laser machining, and introduce the principle of 5-axis CNC laser machining system based on 3-axis galvanometer scanning unit and 2-axis rotation table. We describe the texture mapping approach to controlled distortion rate of 3D texture math model from 2D original bitmap or vector texture applied to high gauss curvature surface. Through a case study of laser marking of a high curvature surface of a mice top case, it shows that the advances in 5-axis laser machining system meet the quality standard of various laser machining.
\end{abstract}

\section{Introduction}

Laser machining (texturing, marking, direct structuring) is a relatively new multi-process technique that has been used for machining 3D curved surfaces. With laser texturing process, it makes more flexible and efficient to create decorative texture on 3D curved surfaces of injection molds so as to improve the surface quality and achieve cosmetic surface of molded plastic parts [1]. Laser making on 3D curved surfaces of products is focused on providing top value solutions and more information for automotive, customer product, gift, electronics and packaging. Laser direct structuring (LDS) on complex 3D carrier is specially used to produce circuit layout directly into the molded plastic part [2].

To begin laser machining, we intend to address the lack of 3D trajectory, which is designed on 3D surface, and through which the focus of the processing laser beam is guided during the 3D machining process. The 3D trajectory is called 3D texture model, 3D marking model and 3D circuit artwork respectively with respect to laser texturing, laser marking and LDS. A planar projection is easy to create 3D trajectory on any 3D curved surface, but it appears correct only from one viewing angle. For example, it is impossible to create a pattern consisting of rectangular on angled surfaces. Figure 1a shows that a number of hexagons from a flat surface are projected on a curved freeform surface, but the projected hexagons on the surface are seriously distorted in geometrical, normal mapped hexagons with controlled distortion rate as shown in Figure $1 \mathrm{~b}$ are expected in the field of 3D laser machining.

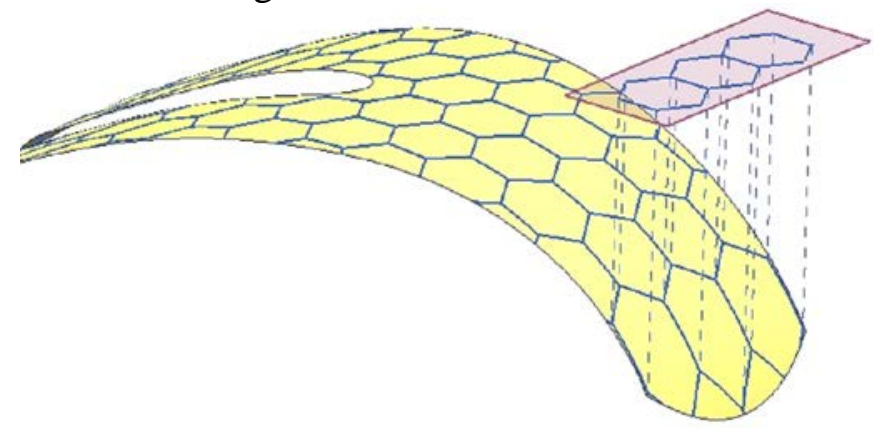

(a) Projection distortion of hexagons

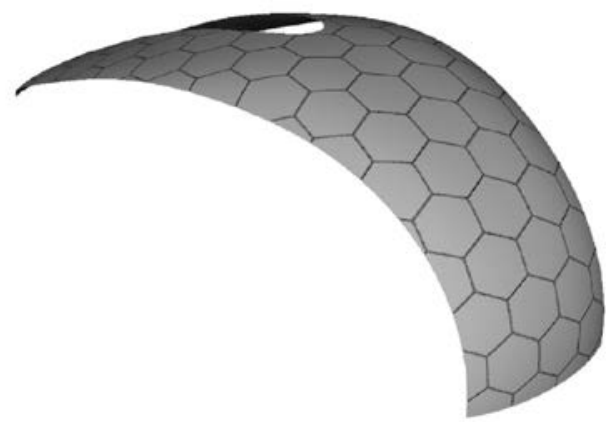

(b) Controlled distortion of mapped hexagons

Figure. 1. 3D hexagon texture achieved by projection and mapping 
In recent years, many research works have been reported in the literature in the area of 3D trajectory of 2.5D or 3D laser machining system. Based on 2.5D layer laser machining, various techniques have been developed to create functional or decorative textures on 3D components [35]. The principle of a 2.5D laser carving and engraving system can be generalized as layered carving. A 3D CAD model achievd by projection was sliced discretely in a certain direction so as to get the profile information of the layer, then $Z$-axis of work table was automatically changed according to the profile information of each layer. A dynamic focusing system, which enables translation of the laser beam along $Z$-axis was used instead of $Z$-axis of work table [6], the motion in the three axes $(X, Y, Z)$ should be synchronized in order to achieve predictable 3D trajectories of the laser beam focus, however, the 3D trajectories were still achieved by projection. Using this simple projection procedure inevitably results in some geometrical distortion of laser marking, a further research was expected to examine projection algorithms, which would minimize geometrical distortion in special 3D case [6].

A few of famous CNC machine manufacturers [7] in the world have developed efficient solution of texture mapping for laser ablating plastic injection mold, they have been using laser beam to replace cutter in their original 5-axis CNC machine to often make the laser beam vertical to the activated surface of mold workpiece, so that any tilted surface of mold can be to laser ablated. Such type of 5-axis CNC machine is much available for laser ablating mold, but not suitable for laser making and laser direct structuring, since its laser machining efficiency is much lower than the requirement of the two laser machining processes.

Texture mapping methodology of laser machining 3D curved surface is a direction to eliminate or minimize distortion of texture model on 3D curved surface. Texture mapping is a term that is used to describe both the actual process of decorating 3D model's surface and the process of fitting a set of $2 \mathrm{D}$ coordinates on a $3 \mathrm{D}$ surface $[1,8]$. A new displacement texture mapping algorithm was developed in [1], in order for the 2D image (texture) is pre-warped to compensate against the distortions caused by the vertical projection. Such similar work has been done in [6]. Benefiting from the experience of our original research of 3D laser mold ablating system in [9], we presented a novel technique of texture mapping on 3D curved surface in [10]. Figure 2 shows the texture mapping process based on parameterization algorithm we developed in [10]. Its process can be applied in 5-axis laser machining for laser texturing of mold, laser making of product and LDS of 3D circuitry.

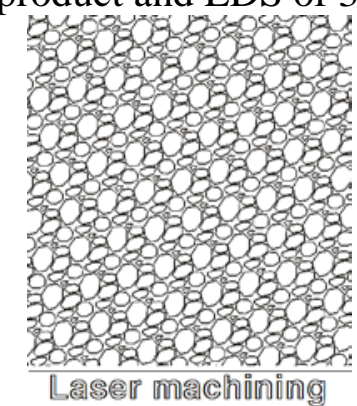

(a) 2D multi-texture

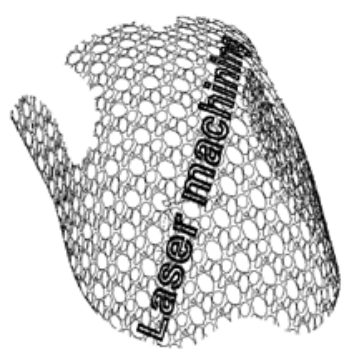

(d) 3D texture model

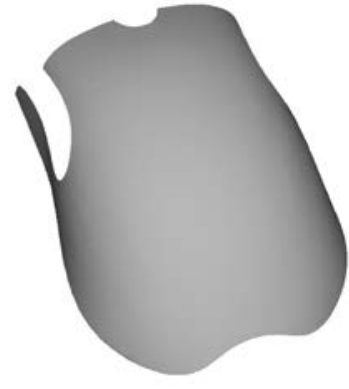

(b) Curved surface

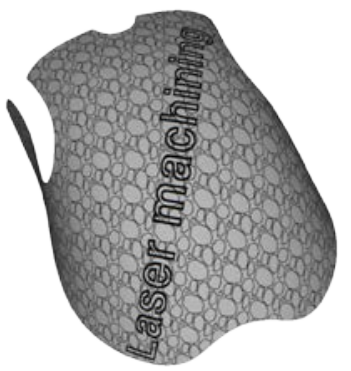

(c) Map texture on surf.

Figure 2. Texture mapping process

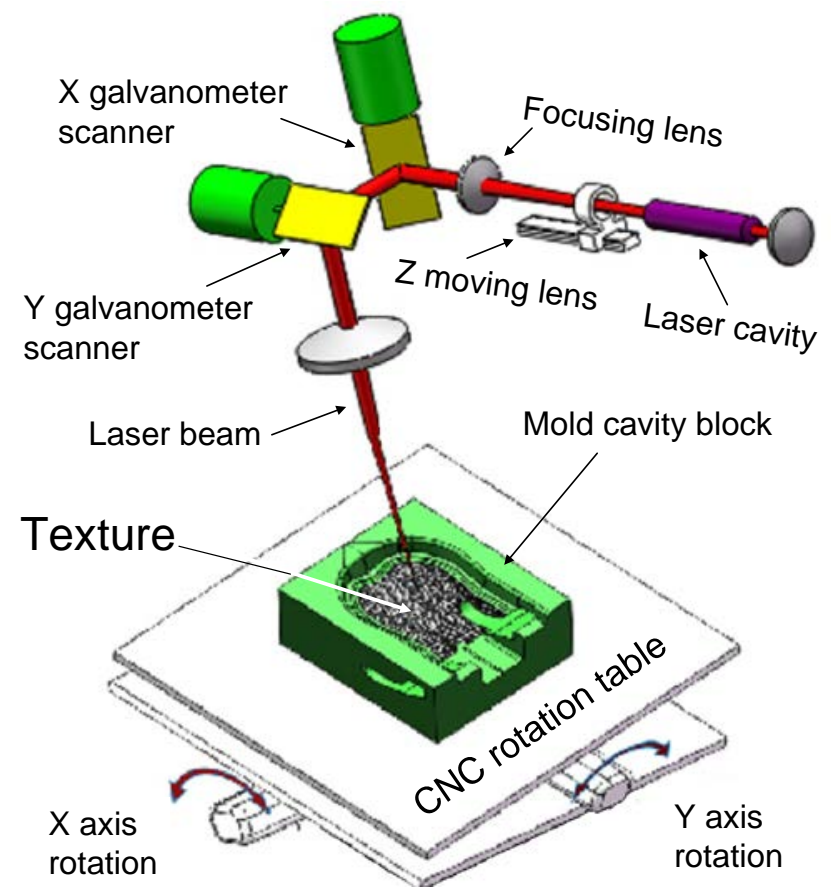

Figure 3. 5-axis laser machining system 


\section{5-axis CNC laser machining system}

5-axis laser machining system is composed of 3-axis optic galvanometer scanning unit and 2axis rotation table. Taking for example laser texturing curved surface of a plastic mold cavity, we present the principle of 5-axis laser machining system as shown in Figure 3. In 3D optic galvanometer scanning unit, laser beam first enters a $\mathrm{Z}$ moving lens and focusing lens. After moving lens, the beam diverges rapidly until it enters one or two focusing lenses. The beam, now converging, passes through and is directed by a set of $X$ and $Y$ mirrors moved by the galvanometer scanners. The orthogonal arrangement of the $X$ and $Y$ mirrors direct the beam down towards and over the length and width of the working field. The focusing height of laser is adjusted by moving $Z$ lens according to the $Z$ coordinates of $3 D$ leather texture model which is the result of computer texture mapping from 2D leather texture. The 3D curved surface is activated by the laser beam, the maximum angle of incidence of the laser beam on the surface to be machined must not be exceeded if safe activation is to be achieved. The angle of incidence is the angle between the orthogonal to the activated surface and the laser beam. Usually the maximum angle of incidence is no bigger than $30^{\circ}$,so that some tilted surfaces are out of its machining scope, therefore a 2-axis rotation table is needed to make sure any tilted surface could be machined through the rotation of table. 5-axis laser machining system, is able to move internal laser beam in three primary axes, designated $\vec{X}, \vec{Y}, \vec{Z}$, and another two axes created by the rotation of the $X$ and $Y$ axes, designed $\hat{X}, \hat{Y}$, therefore five CNC axes are $\vec{X}, \vec{Y}, \vec{Z}, \hat{X}, \hat{Y}$ respectively. The motion in the five axes should be synchronized in order to achieve predictable 3D texture trajectories of the beam focus.

The first aspect of the advances in 5-axis laser machining system is to control 5 axes movement of both laser beam $(\vec{X}, \vec{Y}, \vec{Z})$ and rotation table $(\hat{X}, \vec{Y})$ synchronously. The second is to achieve 3D texture model of high gauss curvature surface in controlled distortion rate. In this research, we create 5-axis laser machining system as shown in Figure 3 to identify and apply the mapped 3D texture model to ablate the texture on the surface of the mold. To guaranty high efficiency of laser marking or laser direct structuring, the whole 3D texture model is often divided into several parts. Before laser machining each part, the $X-Y$ rotation table needs to be run in specified angles accordingly in advance, so that the fast motion in three axes of optic galvanometer scanning unit could not be sacrificed by the slow motion of mechanical $X-Y$ rotation table, the motion in three axes of optic galvanometer scanning unit is synchronized only, and its speed is much faster than the motion speed of mechanical axes in [8].

\section{Texture mapping GUI}

Texture is herein defined as all of what are designed on 3D curved surface, therefore texture includes pattern, logo, image, drawing in laser texturing and marking, it includes circuit artwork in LDS. Customer software is developed in the OpenGL graphical environment allowing basic visualization of acquired 3D surface as well as interactive positioning of the 3D texture model based on the 3D surface. Figure 2 shows that the first step of texture mapping is to design 2D texture, which is either bitmap graphic or vector graphic. We have developed a parameterization algorithm in [10] to meet below requirements for laser machining on any curved freeform surface.

- Single patch free boundary parameterization.

- Guarantee one-one-mapping.

- Offset, scale and orientation control.

- Non-uniform distortion control.

- Distortion should take the angle and stretch into account.

In this research, we develop the graphical user interface (GUI) of generating 3D texture model from either bitmap graphic or vector graphic or both together as shown in Figure 4. The right parts of the GUI show single 2D texture or multi-2D texture, no matter whether those are bitmap graphics or vector graphics. The middle parts present the expansion of 3D curved surface based on parameterization algorithm we developed in [10], and the 2D texture mapped on the expansion 
plane of 3D curved surface. The mapped texture can be scaled, rotated and translated before it is finally mapped on the 3D curved surface. The left parts present the performance of 3D texture model mapped on original 3D curved surface. After removing the original 3D curved surface , the user can achieve the 3D texture individually and save it as STL, OBJ or DXF format file in order for 5-axis laser machining system to machine the 3D curved surface. By selecting major GUI tabs of 5-axis laser machining system, the user can set up measurement and machining process parameters to start laser machining.

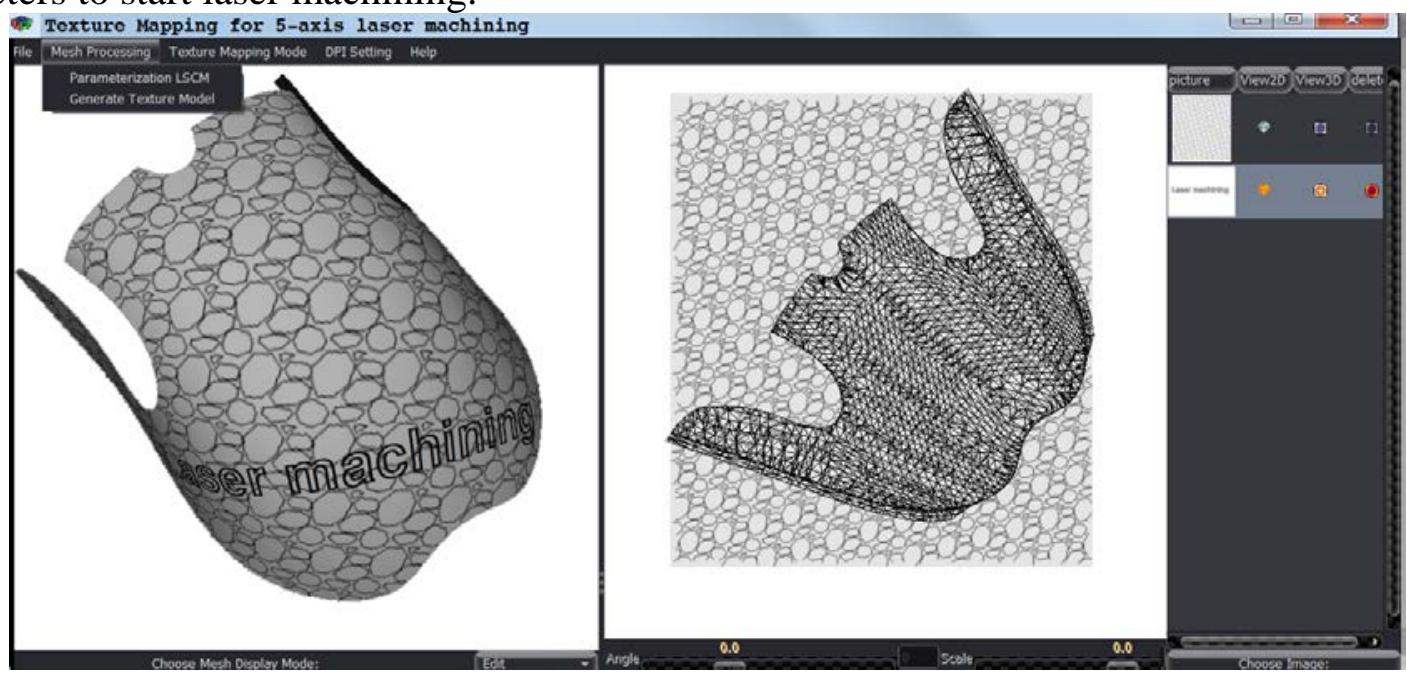

Figure 4. Texture mapping GUI

\section{System implementation}

The distortion rate of marked or ablated texture on 3D curved surface should be controlled in the tolerance of $\pm 5 \%$ according to our investigation on the quality standard of laser machining, which has been defined by a number of manufactures. In order to qualify the accuracy of both the 5-axis laser machining system and the parameterization algorithm performed by the texture mapping GUI, we have mapped texture with a series of hexagons (designed side length $4.00 \mathrm{~mm}$ ) on a high gauss curvature surface of a mice top case, then laser marked the 3D texture model as shown in Figure 5a. Using a CCD projector to measure each side length of all marked hexagon, we have been able to qualify the actual accuracy of 3D texture mapped and marked on the high curvature freeform surface in six different directions for each hexagon. Figure $5 b$ is an example of measurement picture of a hexagon captured by the CCD projector, whose measuring resolution is $0.01 \mathrm{~mm}$ due to its feature of one hundred times magnification as shown in Figure 5c.

It has been found that distortion rate is much low in the Area A, which is almost perpendicular to $Z$ axis as shown in Figure $5 a$. In the Area $B$, where the highest gauss curvature rate exists, the distortion rate is higher that that in Area A.

Figure 6 is the statistical graph with respect to the measurement data of the length of all sides. The measurement date in Area A in Figure 5(a) is distributed into Distribution I in Figure 6, in which measurement data from other area except Area B in Figure 5a is included. However, all of the measurement date in Area B in Figure 5a exactly correspond to Distribution II in Figure 6. 


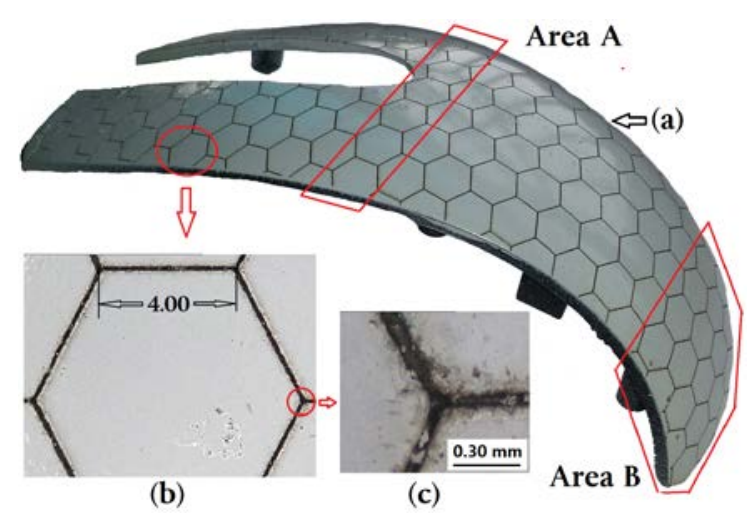

Figure 5. Hexagon texture laser-marked

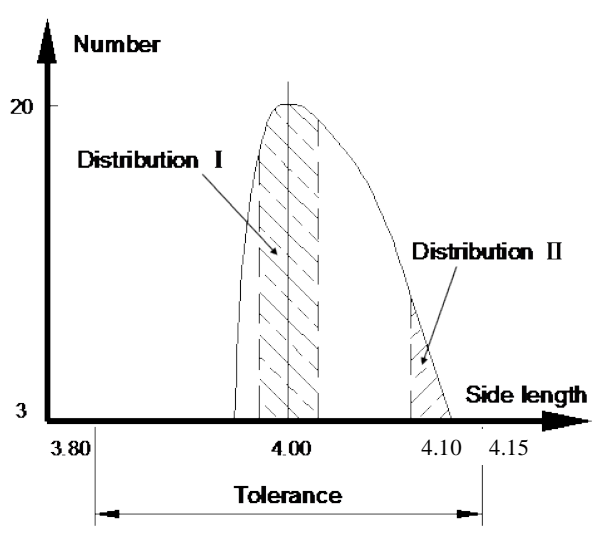

Figure 6. Statistical graph of measurement data

The maximum side length $(4.15 \mathrm{~mm})$ occurs in Distribution II, so the maximum distortion rate is $3.75 \%$ only, and is acceptable according to the quality standard $( \pm 5 \%)$. From Figure 6 , it is easily found that distortion of laser machining often happens in the areas of high gauss curvature surface, the root cause is from both the deviation of laser beam motion and the inevitable warp of texture mapping performed by the parameterization algorithm.

\section{Conclusion}

Laser machining 3D high curvature surface is a big challenge in the field of laser manufacturing industry. Targeting the challenge, this research has developed a new innovation and solution by which laser machining on high curvature surface is available and high efficient. The advances in 5axis laser machining system apply just enough processes to ensure laser machining quality since the system implementation has qualified the synthesized accuracy of both 5-axias laser machining system and parameterization algorithm performance. For further research, both algorithms regarding to 5-axis synchronized motion and parameterization process of texture mapping are expected to get improved in order to make least amount of distortion of laser machining on high curvature surface.

\section{Acknowledgements}

This work is supported by Guangdong Science and Technology Plan (A2015A050502006) in China, Shenzhen Science and Technology Plan (ZJHZ20150316112419786) in China, Shenzhen Science and Technology Plan (JCYJ20150417094158016) in China. The authors are greatly indebted to many field engineers for helping conducting lots of in-situ test.

\section{Reference}

[1] Henrikki Pantsar, Reino Ruusu, Jansson, A. Advances in 3D laser processing in mold technology. In Proceedings of the 25th International Congress on Applications of Laser and ElectroOptics. ICALEO 2006.

[2] Gries Doug. Laser direct structuring creates 3D integrated circuit. Laser Focus World 2010; 46(10):54-63

[3] A Bereczki, GA Cirino and SP Morato. Tridimensional laser engraving of industrial injection moulds for fresnel surface generation, Ann Opt 2006.

[4] C Wang and X Zeng. Study of laser carving three-dimensional structures on ceramics: quality controlling and mechanisms. Opt Laser Technol 2007;39:1400-1405.

[5] MSC Williams, Method of three dimensional laser engraving. WIPO, Pub. No.: WO/2000/074891. 
[6] Janez Diaci, Drago Bračun. Rapid and flexible laser marking and engraving of tilted and curved surfaces. Optics and Lasers in Engineering 2011;49(2):195-199.

[7] Agile Chamiller Laser ablation manuals; 2017< http://www.gfms.com />

[8] Eugene Zhang, Konstantin Mischaikow, and Greg Turk. Feature-based surface parameterization and texture mapping. ACM Trans. Graph.2005;24:1-27

[9] Yong-quan Zhou, Yi-feng Wu, Sheng-yu Zhao. Key Technology Study of Laser Texturing Machine of Plastic Mould. China Plastics Industry 2011;39(2):56-62

[10] Anjie Lu, Yongquan Zhou, Wanjun Zhang, Songling Zhang. Texture mapping techniques of 3D laser marking. Journal of Shenzhen Institute of Information Technology 2013;11(1):25-28 